\title{
The Michigan Infrared Combiner (MIRC): IR imaging with the CHARA Array
}

John D. Monnier, Jean-Philippe Berger, Rafael MillanGabet, Theo A. ten Brummelaar

John D. Monnier, Jean-Philippe Berger, Rafael Millan-Gabet, Theo A. ten Brummelaar, "The Michigan Infrared Combiner (MIRC): IR imaging with the CHARA Array," Proc. SPIE 5491, New Frontiers in Stellar Interferometry, (20 October 2004); doi: 10.1117/12.550804

Event: SPIE Astronomical Telescopes + Instrumentation, 2004, Glasgow, United Kingdom 


\title{
The Michigan Infrared Combiner (MIRC): Infrared imaging with the CHARA Array
}

\author{
John D. Monnier ${ }^{a}$, Jean-Philippe Berger ${ }^{b}$, Rafael Millan-Gabet ${ }^{c}$, and Theo ten Brummelaar ${ }^{d}$ \\ ${ }^{a}$ University of Michigan (Astronomy), 500 Church St, Ann Arbor, MI 48104 USA; \\ ${ }^{b}$ Lab. d'Astrophysique de l'Observatoire de Grenoble, Grenoble, France; \\ ${ }^{c}$ Michelson Science Center (Caltech), Pasadena, CA, USA; \\ ${ }^{d}$ Georgia State University CHARA Array, Mt. Wilson, CA, USA
}

\begin{abstract}
We present the design of the Michigan Infra-Red Combiner (MIRC). MIRC is planned for deployment at the Georgia State University CHARA array to simultaneously combine all six telescope beams in an image-plane combiner. The novel design incorporates spatial-filtering with single-mode fiber optics, a synthetic (densified) pupil, and a low-resolution spectrometer to allow good calibration and efficient aperture synthesis imaging in the near-infrared. In addition, the focalization and spectrometer optics can accommodate an integrated optics component with minimal re-alignment. The MIRC concept can be scaled-up for interferometer arrays with more telescopes.
\end{abstract}

Keywords: infrared, interferometry

\section{INTRODUCTION}

The Georgia State University (GSU) Center for High Angular Resolution Astronomy (CHARA) interferometer is a 6-telescope optical array located on Mt. Wilson ${ }^{1}$. In a collaboration with GSU and Grenoble Observatory, the University of Michigan has begun work on the Michigan Infra-Red Combiner (MIRC) to act as an "imaging" infrared combiner to combine all six telescopes together to allow true interferometric imaging with CHARA from 1.45-2.5 $\mu \mathrm{m}$ with $\sim 1$ milliarcsecond resolution.

\section{COMBINER FEATURES}

\subsection{Overview}

In order to pursue the twin goals of efficient imaging and high-precision calibration, we have the following design principles for the MIRC combiner:

- Image Plane Combination: 6 telescopes, 15 visibilities, 20 closure phases simultaneously (and measurements of closure amplitudes for the first time)

- 1.45-2.5 microns ( $\mathrm{H}$ and $\mathrm{K}$ band separately, simultaneously if possible)

- Low-resolution spectroscopy, R 100 to 300

- Spatial filtering (with photometric monitoring eventually) using single-mode fibers

- Easy integration with integrated optics combiners

- Straight-forward integration with a separate fringe tracker 


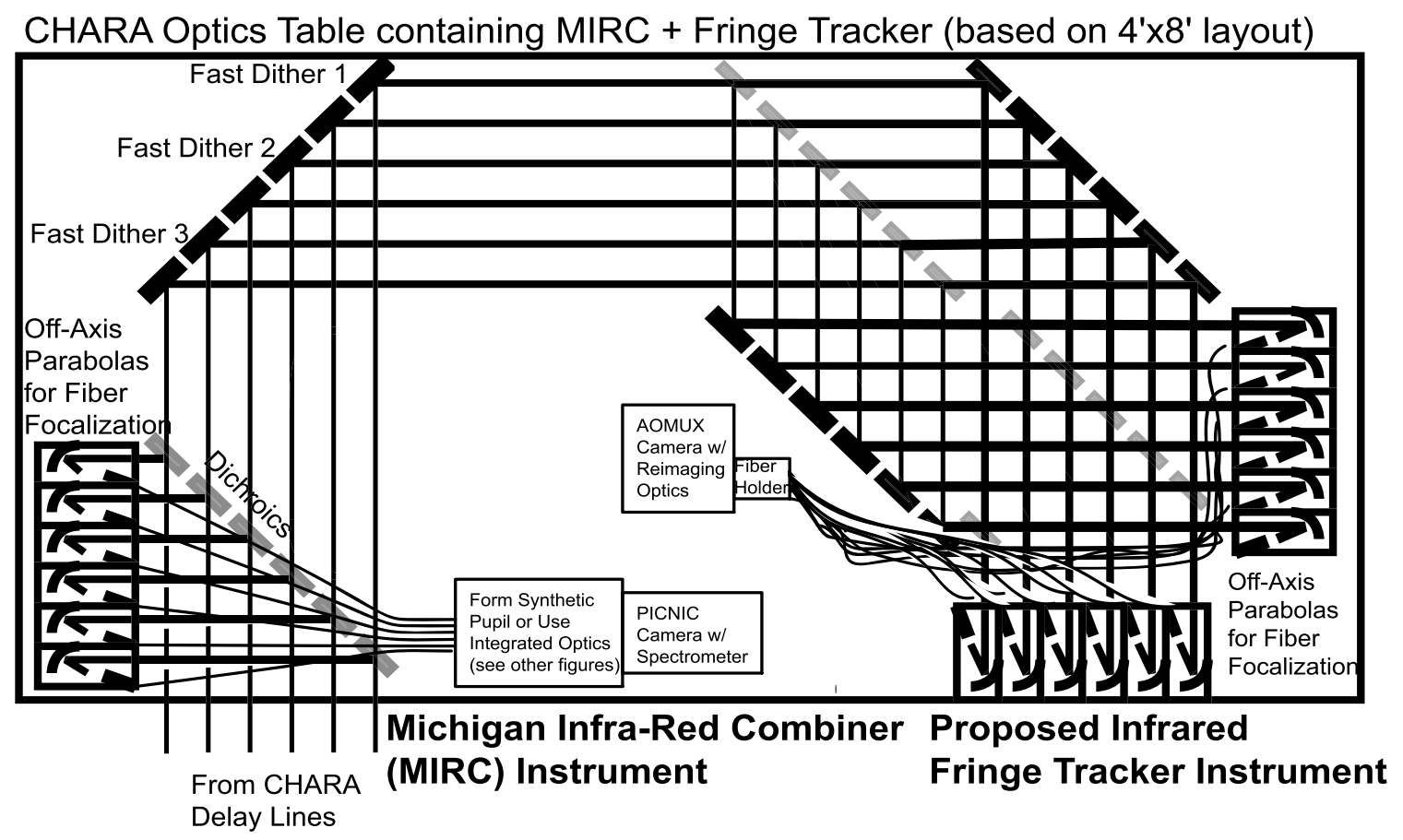

Figure 1. The beams from the CHARA telescopes are reflected using dichroic beamsplitters and focused into single-mode fibers. The fibers are then brought back together to form a one-dimensional non-redundant array. This synthetic pupil is dispersed by a grism before being imaged on a PICNIC array camera. A beam-combining solution employing an integrated optic component, instead of a synthetic pupil, is also being explored. The right part of this optical table sketch shows a proposed fringe tracker layout for future integration with MIRC.

Image plane combination is being pursued, to allow simultaneous measurements of all 15 available baselines and 20 closure phases in the full 6-telescope CHARA array. The beams from all telescopes will be arranged in a linear non-redundant pattern and then imaged onto a slit to be analyzed using an infrared spectrograph at the backend. In order to allow precision calibration of visibility amplitudes and closure phases, the synthetic pupil will be formed using light transported and spatially-filtered by single-mode fibers for optical stability. ${ }^{2}$ A lowresolution grism (or other dispersive element) on the output ( $\mathrm{R} \sim 100$ to 300 ) will mitigate dispersion problems and bandwidth-smearing of data (especially important with the long CHARA baselines). This proposal is very similar to the recommended designs evaluated by Theo ten Brummelaar and Steve Ridgway in early CHARA technical memos, ${ }^{3,4}$ for example appendices $\mathrm{K}$ and $\mathrm{L}$.

While these design features are necessary for good astrophysics, they are achieved at great expense in sensitivity. Detector readnoise will be the dominating noise source under almost all observing conditions, because both all-in-one beam combination and the dispersive element spread out the available light across many pixels. We note that as detectors improve, these deficiencies become less important and approach the same sensitivity as pair-wise, broadband schemes.

We ascribe to the philosophy that mature interferometers will all have dedicated fringe tracking systems, just like large telescopes all have adaptive optics. By separating out the light for fringe tracking from the light used for science, each combiner can be separately optimized. This is critical, since fringe trackers need lots of photons but good calibration is not needed. MIRC was designed with the assumption that an infrared fringe tracker would be built to recover the sensitivity lost ( $\sim$ magnitudes) in pursuing good calibration and powerful imaging capabilities - a fringe tracker will be integral to MIRC's ability to observe a wide-range of targets.

Further author information: J.D.M.: E-mail: monnier@umich.edu; J.-P.B.: E-mail: Jean-Philippe.Berger@obs.ujfgrenoble.fr; R.M.G.: E-mail: rafael@ipac.caltech.edu; T.tB.: E-mail: theo@chara.gsu.edu 


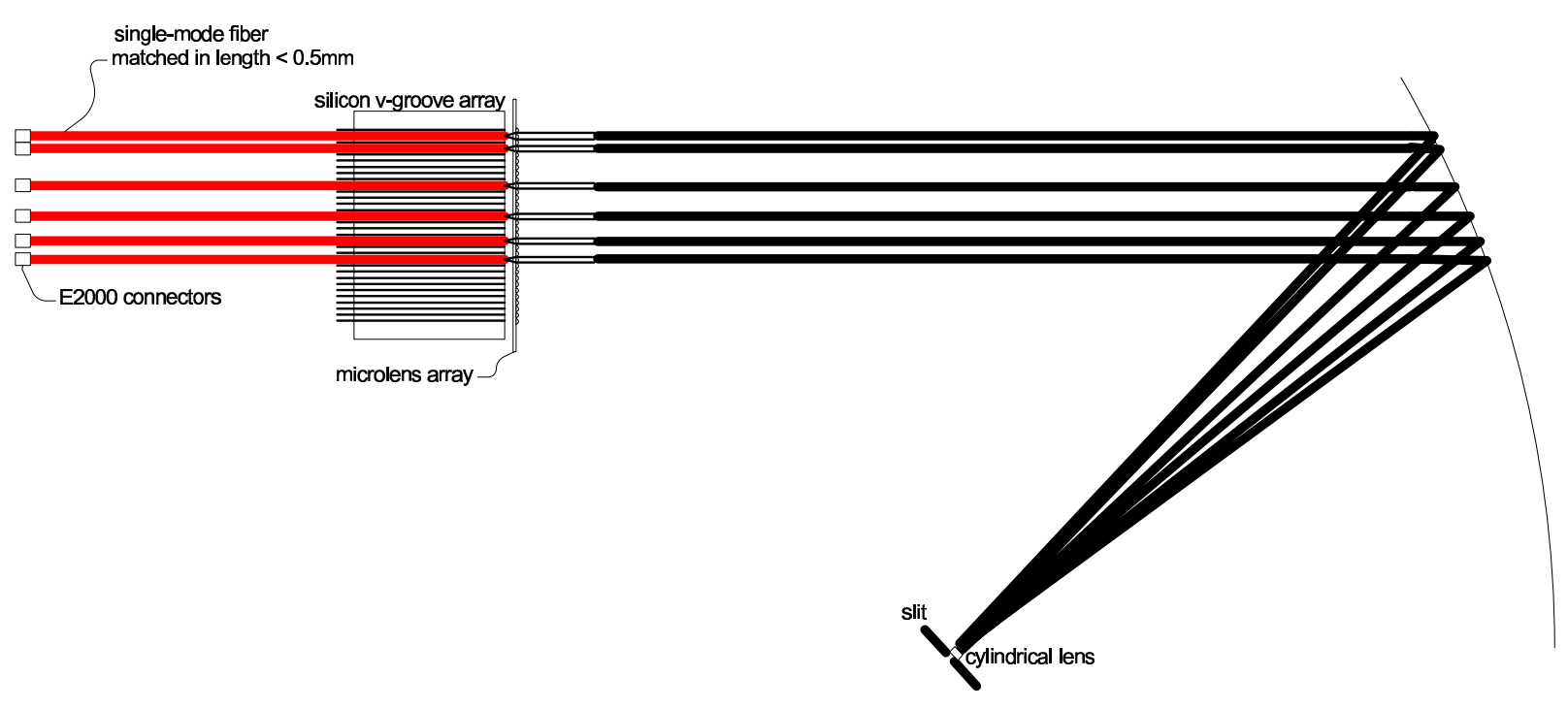

Figure 2. This figure shows a more detailed schematic of the fiber arrangement and combination scheme in the MIRC combiner. Fibers are aligned in non-redundant pattern in a silicon V-groove array and aligned with a micro-lens array. The miniature collimated beams are focused onto a slit with the help of a cylindrical lens.

This "science combiner with separate fringe tracker" design has been developed over many years, and was probably first best expressed by the CHARA team in their original NSF proposal. ${ }^{5}$ This represents a major departure from current approaches at the "first generation" imaging interferometers COAST and NPOI. We note that the European Southern Observatory Very Large Telescope Interferometer (ESO-VLTI) has adopted a similar architecture for the AMBER instrument, ${ }^{6}$ which also will require a separate fringe tracker unit (FINITO ${ }^{7}$ ) to achieve reasonable sensitivity at high spectral resolution.

\subsection{Unique Science Capabilities}

We would like to highlight two key science programs that we are most interested in pursuing using the particular advantages of this kind of combiner.

- With sub-AU resolution, current interferometric arrays can directly probe the inner accretion disks of young stellar objects. The physical conditions of these regions are poorly understood, ${ }^{8,9}$ yet are fundamental ingredients in understanding the process of planet formation as well as the inward migration of gas giant planets formed in the outer disk. Recent work ${ }^{10}$ suggests further that a "puffed-up" inner disk can shadow the outer disk with potentially dramatic effects on both our interpretation of spectral energy distributions and the pre-conditions for planet formation. CHARA with the MIRC combiner will have unprecedented imaging capability ( 15 baselines and 20 closure phases simultaneously) with clear advantage for imaging circumstellar emission compared to combination schemes like at COAST which only combine 3 or 4 together at once, or VLTI which will only have 3-telescope combination in the near future.

- Detecting faint companions to bright stars (i.e., planets). By synthesizing a pupil and dispersing the light after spatial filtering, one can maximize mechanical and optical stability, aspiring to achieve millidegree precision on closure phase measurements of bright targets. "Hot jupiters" can be detected via the closure phase signal, ${ }^{11,12}$ a detection channel not currently being widely pursued that has many inherent calibration advantages over so-called "differential phase" (photocenter shift) methods. Lastly, by using single-mode fibers to combine 4 or more telescope simultaneously, we are able to measure the closure amplitude for the first time in optical interferometry - a potentially valuable quantity ${ }^{13}$ for imaging and planet detection programs. 


\section{DESIGN}

\subsection{Overview}

A preliminary (physical) layout of the MIRC instrument can be seen in Figure 1 (left side of optics table), where it is shown already integrated with a fringe tracker (yet to be funded). All the basic combiner features, as described above in $\S 2.1$ are present. The plan is for the combiner to be completely developed and tested at the University of Michigan, and then transported to CHARA for integration. In this section we will describe in more detail how the MIRC design goals are to be actually implemented.

\subsection{Focalization Modules}

Using the beam switchyard capabilities of CHARA, a subset of 6 telescope pairs will be used to "phase-up" the array, allowing longer integrations on the imaging combiner (MIRC). Dichroic beamsplitters will pick off science light and pass the remaining spectum to the fringe tracker unit (not to be discussed in detail here). Science light reflected from the dichroics will encounter the MIRC "focalization modules."

Each focalization module will couple one CHARA telescope beam (compressed to $19 \mathrm{~mm}$ here) into a single mode fiber. We are optimizing the design for $\mathrm{NA}=0.17$ fibers for light between $1.5-2.4 \mu \mathrm{m}$, assuming good seeing conditions. ${ }^{14,15}$ We expect to purchase diamond-turned off-axis parabolas with $\lambda / 10 \mathrm{P}-\mathrm{V}$ (at HeNe) surface quality with effective focal length of $60 \mathrm{~mm}$, for $\mathrm{f} / 3$ operation - detailed studies of optimal f/\# are still in progress.

For optimal coupling, the position of the fiber tip must be adjustable at the sub-micron level. Use of piezoelectric stacks are expensive and we are investigating less-expensive alternatives, such as stepper motorbased flexure stages with leverage to reduce backlash to $<0.2 \mu \mathrm{m}$ level (e.g., see company literature of Luminos). We have adopted the E2000 connector style for the fibers, following the standard set by the FLUOR group ${ }^{16}$ in Paris and the IONIC group ${ }^{17}$ in Grenoble. This will allow our system to be easily used for integrated optics ${ }^{18}$ or future fiber combiners.

\subsection{Combiner}

Figure 2 shows our initial design for the combiner. The fibers containing starlight are custom-made to allow singlemode propagation from 1.5 to $2.4 \mu \mathrm{m}$. Currently, we have acquired polarization-maintaining fiber (Highwave) with these properties (NA 0.18) made from low-OH silica to achieve reasonable (though not ideal) transmission through K-band. We are also in the process of acquiring (circular) Fluoride fibers with similar waveguide properties but with higher transmission.

The fibers will be arranged precisely in a silicon V-groove array, as is common for integrated optics applications. ${ }^{18}$ The spacing of the fibers will not be uniform, but rather will be arranged in a non-redundant pattern so that every fiber pair will have a unique spacing. One interesting arrangement is shown in Figure 2: spacings of 2-6-5-4-3. By populating the V-groove with additional fibers, we can explore different combination strategies simply by plugging in different fibers into the focalization modules. The light beams diverge from the fiber tips and are collimated by a microlens array. Commercial arrays (SUSS-microoptics) are available in fused silica and silicon, however the standard AR-coatings are not ideal for astronomy (covering only the telecom range, 1.25-1.65 $\mu \mathrm{m})$. Custom microlens fabrication using Infrasil would improve transmission and also the microlens focal lengths could be matched optimally to the fibers being used. We note that the dispersion in the glass make the optimal microlens focus wavelength-dependent, and in $\S 4$ we present a calculation that explores this problem.

Lastly, the collimated beams are focused using a long focal length off-axis parabola. The large f/\# achieves the high magnification needed to see the finest fringes on the detector (PICNIC detector with $40 \mu \mathrm{m}$ square pixels). Since the interferometric information is limited to a single dimension, we can use a cylindrical lens to focus the light in the spectral-dimension to a tighter focus (see Figure 4). This also allows us to place a slit at the focus of a spectrograph and to carry-out spectroscopy.

Unfortunately, our current design does not incorporate monitoring of the fiber photometric signals. ${ }^{2}$ This is a potentially very serious limitation to the achiavable calibration precision of visibility amplitudes. However, 


\section{ZEMAX Ray Tracing}

TOP VIEW

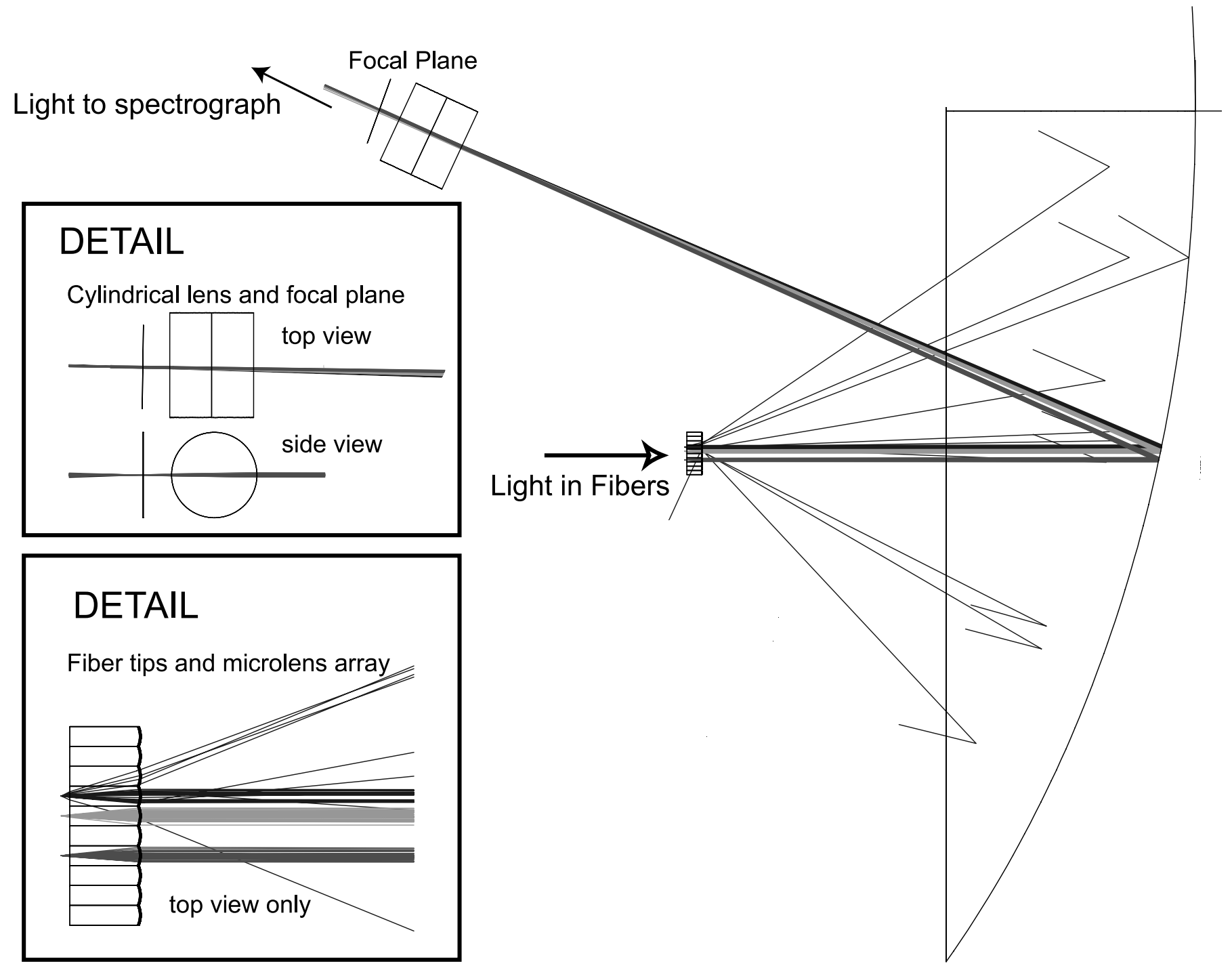

Figure 3.

This figure shows a ZEMAX optical ray tracing of the MIRC combiner, realized with only 3 fibers instead of 6 . Insets show DETAIL of the microlens array collimation of the beams and the anamorphic optics used before the first focal plane. 

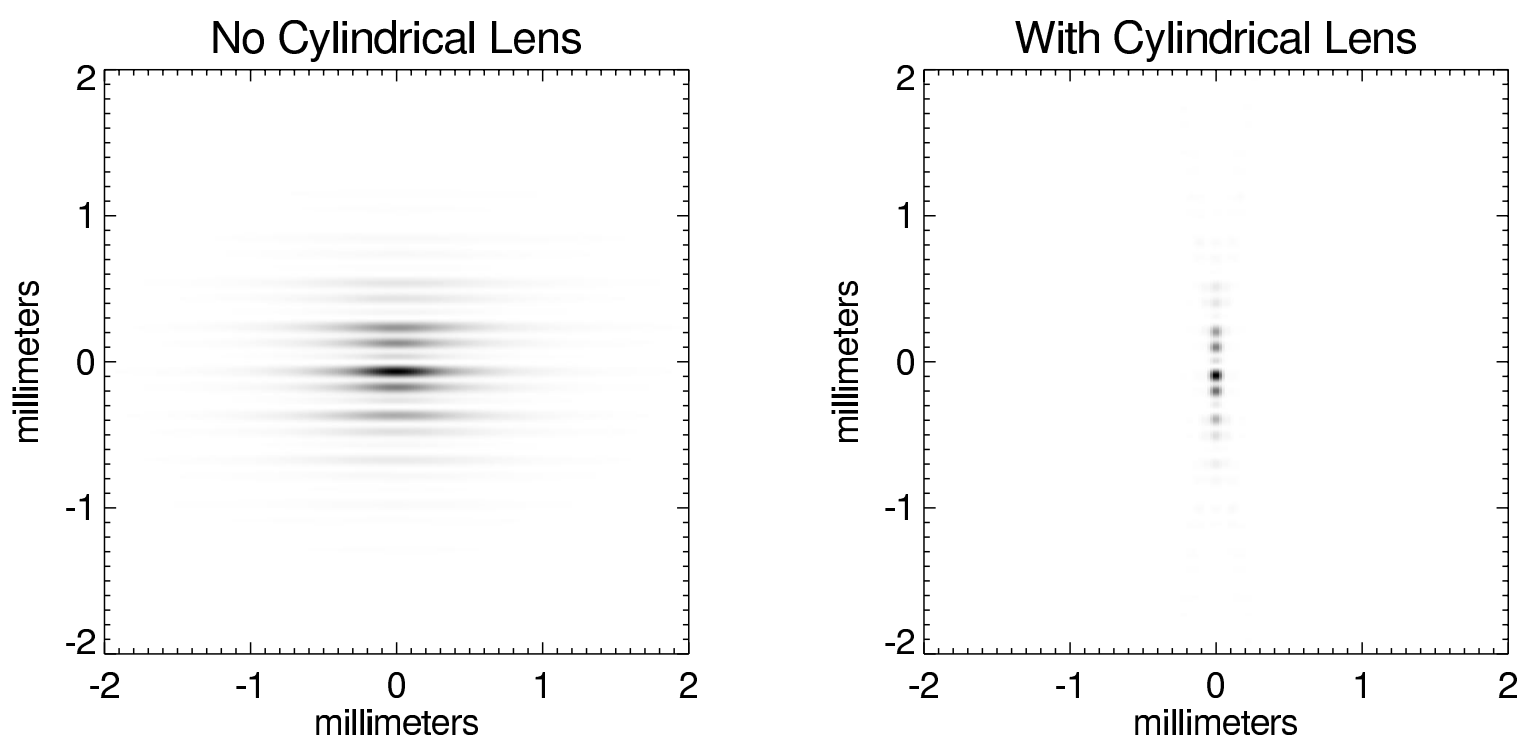

Figure 4. This figure shows the light intensity (linear scale) at the focal plane shown in Figure 3 for $1.65 \mu \mathrm{m}$ light. The left panel shows the pattern when there is no lens to concentrate the light. The right panel show how a simple cylindrical lens concentrates the light into a line suitable for injection into a spectrograph.

the Palomar Testbed and Keck Interferometer combiners have shown that reliable visibility calibration of $\Delta V<$ $5 \%$ (adequate for many applications) is achievable with a comparable method of calibration. ${ }^{19,20}$ We are investigating methods to pickoff light from the collimated beams before combination, but the most straightforward implementation involves a second IR camera which we do not yet possess.

\subsection{Spectrometer}

The spectrometer currently planned will have options to use a (warm) non-deviating prism-pair for R 20 spectral resolution or one of two cold grisms for $\mathrm{R} \sim 100$ or 300 . The spectrometer optics have not been fabricated, but are currently expected to be $\mathrm{f} / 4$ achromatic doublets based on a design used by the Keck Interferometer.

The detector will be a PICNIC array detector, sensitive from 1 to $2.5 \mu \mathrm{m}$. Limiting the wavelength coverage to $2.5 \mu \mathrm{m}$ saves complexity in the combiner, since all of optics will not need to be cooled. This combiner will require reading-out a relatively large area $(\sim 50 \times 120$ pixels $)$ at high rates $(\gtrsim 20 \mathrm{~Hz})$ with low read noise $(\sim 15$ $\mathrm{e}^{-}$). We are using the cold fanout board and warm analog amplifiers from IR Labs and using high-speed digital electronics (including ADCs) from Astronomical Research Cameras (Bob Leach).

In order to have full control of the data acquisition and realtime feedback to the CHARA interferometer for fringe tracking, the camera will be interfaced under RT-linux (v3.2 with Red Hat 9.0 and its 2.4.21 kernel). Using the fastest fiber interface board offered by Leach (12.5 Mpixels/sec), we plan to readout the PICNIC array at maximum data rates, retaining every non-destructive read. Custom timing patterns will be written to maximize signal-to-noise ratio and to allow sophisticated data analysis, such as employing variable coherent integration times in post-processing.

\section{SIMULATED PERFORMANCE}

This optical design has been simulated using ZEMAX optical design software. Figure 3 shows both the overall geometry (similar to Figure 2) as well as DETAIL insets of the microlens array and focal plane after the cylindrical lens. We only have simulated 3 -fibers in this case for simplicity. Figure 4 shows a sample ZEMAX calculation of the coherent radiance at the focal plane with and without the cylindrical lens, showing the strong improvement in focalization for the spectral direction. 

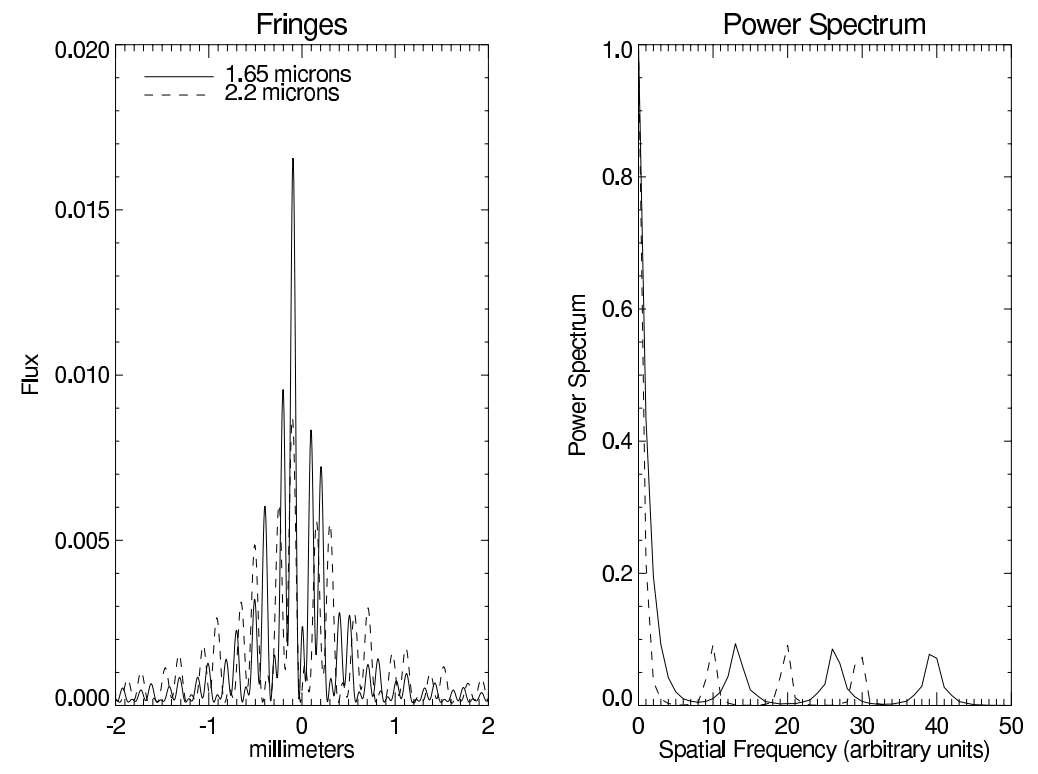

Figure 5. The left panel shows the intensity for $1.65 \mu \mathrm{m}$ and $2.2 \mu \mathrm{m}$ fringes for a fixed focus of microlens (was not "refocused" when switching between wavelengths). The right panel shows the corresponding power spectra with well-isolated peaks for each baseline. Interestingly, the optical performance for both $\mathrm{H}$ and $\mathrm{K}$ band observing appears acceptable without requiring re-adjustment of the microlens focus.

ZEMAX also permits us to test the chromatic dependence of the optical design. Figure 5 show the fringe intensity and the power spectrum for $1.65 \mu \mathrm{m}$ and $2.2 \mu \mathrm{m}$ performance without changing optical configuration. Despite dispersion in the fused silica microlens and cylindrical lens, the fringes are still well-separated in Fourier Space and suggests that this combiner will allow broadband operation $(1.5-2.2 \mu \mathrm{m})$ operation without changing optical alignment.

\section{CURRENT STATUS}

Most of the long lead-time items have already been procured for this project, including the camera hardware and custom single-mode fiber.

In 2004 May, we carried out the first cooldown of the PICNIC detector with the new (IRLabs) dewar and (Leach) digital electronics using Linux. Figure 6 shows one of the first PICNIC images using a flashlight through a pinhole. No attempt was made to flat-field, sky-subtract, or optimize timing patterns or bias voltages. We are in the process of optimizing and characterizing the noise properties of the array.

\section{FUTURE WORK}

The MIRC combiner using all 6 telescopes should be tested and first used for science at CHARA in 2005, assuming no serious problems are uncovered in lab testing. Originally, we had anticipated an earlier test date of a prototype. However, since all the beams are aligned on the V-groove array, there is little advantage to testing a 3-telescope prototype compared to a 6 -telescope prototype.

In parallel, an integrated optics combiner in Grenoble is being pursued by Dr. Berger and his group. The MIRC spectrometer and focalization optics are being fabricated specifically to be compatible with the integrated optics hardware in development in France. We anticipate testing of this combiner to proceed on a similar time scale.

Lastly, we emphasize that the MIRC combiner will only realize its full potential when paired with a fringe tracker. Unfortunately, the MIRC combiner, as any spectrograph, will have low throughput and many choices 


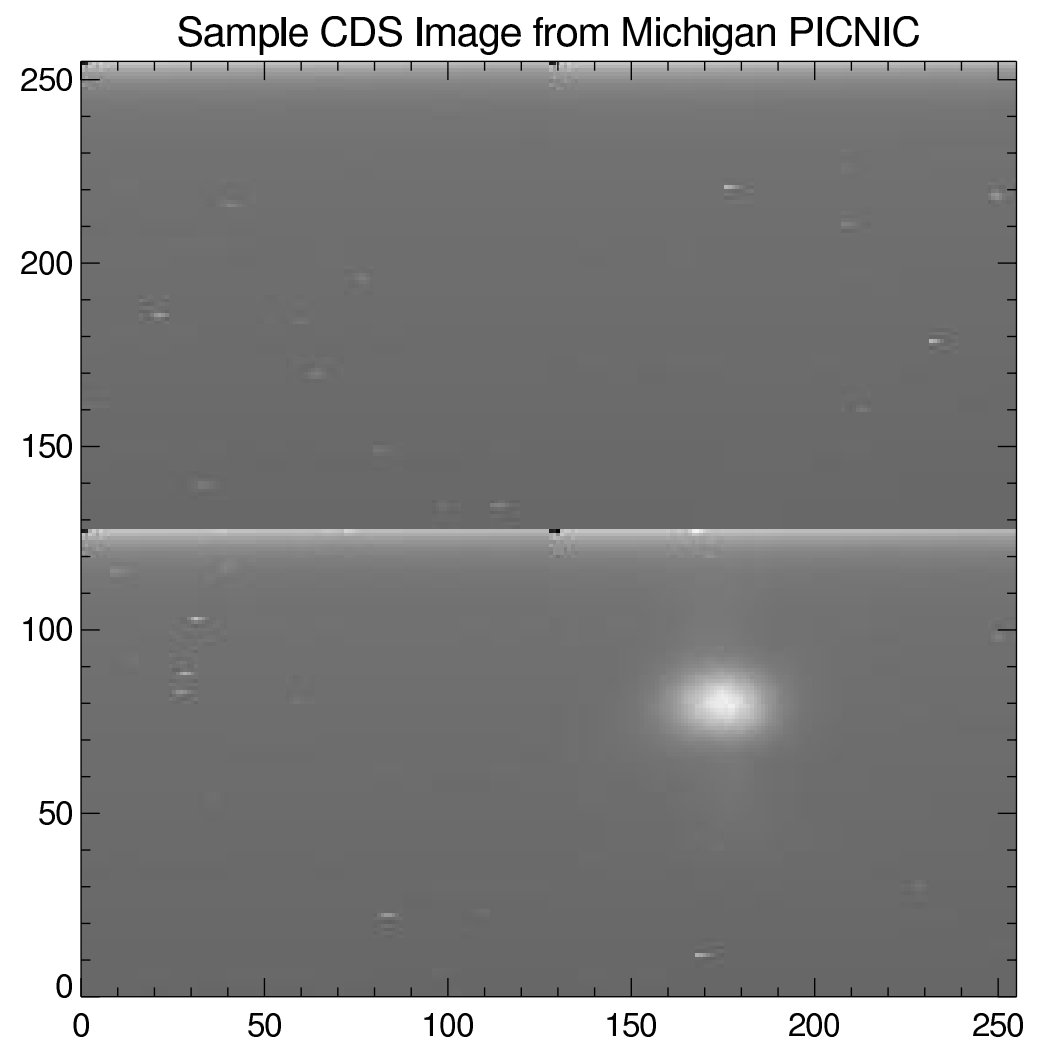

Figure 6. Sample full-frame image (using correlated double-sampling) from the first cooldown of Michigan PICNIC chip (E. Pedretti, R. Millan-Gabet, J. Monnier). Many artifacts are still present because bias and reset voltages have not been optimized. The bright feature in bottom right quadrant is the image of a flashlight through a pinhole.

we have made to improve calibrations have come at the expense of sensitivity. We look forward to implementing infrared fringe tracking soon with MIRC.

\section{CONCLUSION}

We have presented the design for the MIRC combiner for the CHARA interferometer. This combiner is forwardlooking and has the potential to revolutionize the way optical interferometry is done. By leveraging advances in the telecommunication industry for astronomy, we have created a practical design optimized for imaging and calibration that can be easily scaled-up for future interferometer arrays with more than 6 telescopes. In addition, as readnoise becomes smaller for new infrared detectors, this designer concept becomes even more attractive compared to alternative designs.

\section{ACKNOWLEDGMENTS}

This design concept is the result of conversations with many interferometrists, and the authors have appreciated their input. We have tried to take advantage of lessons learned in the fabrication and use of recent combiners, in particular from NPOI, COAST, AMBER (VLTI) and FATCAT (Keck Interferometer). We specially thank G. Vasisht and G. Perrin for specific help with regard to IR camera and fiber issues. We also acknowledge the important help of Ettore Pedretti, who has been working on the PICNIC camera development during his Michelson Postdoctoral Fellowship at University of Michigan. Lastly, we acknowledge the State of George, the National Science Foundation, the W.M. Keck Foundation, and the Lucille and David Packard Foundation for funding the construction of the CHARA array; array operations are being supported by Georgia State University and NSF-AST 0307562. 


\section{REFERENCES}

1. T. A. ten Brummelaar, H. A. McAlister, S. T. Ridgway, N. H. Turner, L. Sturmann, J. Sturmann, W. G. Bagnuolo, and M. A. Shure, "An Update of the CHARA Array," in Interferometry for Optical Astronomy II. Edited by Wesley A. Traub. Proceedings of the SPIE, Volume 4838, pp. 69-78 (2003)., pp. 69-78, Feb. 2003.

2. V. Coudé du Foresto, S. Ridgway, and J.-M. Mariotti, "Deriving object visibilities from interferograms obtained with a fiber stellar interferometer," A\&AS 121, pp. 379-392, Feb. 1997.

3. T. ten Brummelaar, "Visible light imaging," Tech. Rep. Appendix-K, CHARA Array, Georgia State University, Atlanta, GA, 1993.

4. S. Ridgway, "Infrared beam combining and detection," Tech. Rep. Appendix-L, CHARA Array, Georgia State University, Atlanta, GA, 1993.

5. T. ten Brummelaar, "Visible light imaging," Tech. Rep. Appendix-I, CHARA Array, Georgia State University, Atlanta, GA, 1993.

6. F. Malbet, R. G. Petrov, I. Tallon-Bosc, K. Hofmann, D. Mourard, T. Forveille, F. Cassaing, F. Reynaud, M. Tallon, P. Mege, and K. Rousselet-Perraut, "System analysis of the AMBER instrument on VLTI," in Interferometry for Optical Astronomy II. Edited by Wesley A. Traub . Proceedings of the SPIE, Volume 4838, pp. 1163-1170 (2003)., pp. 1163-1170, Feb. 2003.

7. M. Gai, L. Corcione, L. Delatge, D. Gardiol, A. Gennai, M. G. Lattanzi, D. Loreggia, G. Massone, S. Menardi, and F. Reynaud, "The FINITO Fringe Sensor for VLTI," in Beyond conventional adaptive optics. ESO Conference and Workshop Proceedings, Vol. 58, ISBN 3923524617, pp. 329-+, 2002.

8. R. Millan-Gabet, F. P. Schloerb, and W. A. Traub, "Spatially Resolved Circumstellar Structure of Herbig AE/BE Stars in the Near-Infrared," ApJ 546, pp. 358-381, Jan. 2001.

9. J. D. Monnier and R. Millan-Gabet, "On the Interferometric Sizes of Young Stellar Objects," ApJ 579, pp. 694-698, Nov. 2002.

10. C. P. Dullemond, C. Dominik, and A. Natta, "Passive Irradiated Circumstellar Disks with an Inner Hole," ApJ 560, pp. 957-969, Oct. 2001.

11. D. Segransan, "Does the differential phase closure allow to detect photons from hot jupiters?," in SF2A-2002: Semaine de l'Astrophysique Francaise, June 2002.

12. J. Monnier, "Astrophysics with closure phases," in Eurowinter School: Observing with the Very Large Telescope Interferometer, 2002.

13. A. C. S. Readhead, R. C. Walker, T. J. Pearson, and M. H. Cohen, "Mapping radio sources with uncalibrated visibility data," Nature 285, pp. 137-140, May 1980.

14. S. Shaklan and F. Roddier, "Coupling starlight into single-mode fiber optics," Appl. Opt. 27, pp. 2334-2338, June 1988.

15. S. B. Shaklan, "Multiple Beam Correlation Using Single-Mode Fiber Optics with Application to Interferometric Imaging," Ph.D. Thesis , 1989.

16. V. Coude du Foresto and S. T. Ridgway, "Fluor - a Stellar Interferometer Using Single-Mode Fibers," in High-Resolution Imaging by Interferometry, pp. 731-+, 1992.

17. P. Kern, F. Malbet, I. Schanen-Duport, and P. Benech, "Integrated optics single-mode interferometric beam combiner for near infrared astronomy," in Integrated Optics for Astronomical Interferometry (1996 Oct 15-16, Grenoble, France), pp. 195-+, 1997.

18. J. P. Berger, P. Haguenauer, P. Kern, K. Perraut, F. Malbet, I. Schanen, M. Severi, R. Millan-Gabet, and W. Traub, "Integrated optics for astronomical interferometry. IV. First measurements of stars," A\&A 376, pp. L31-L34, Sept. 2001.

19. M. M. Colavita, "Fringe Visibility Estimators for the Palomar Testbed Interferometer," PASP 111, pp. 111117, Jan. 1999.

20. M. Colavita and sixty-one colleagues, "Observations of DG Tauri with the Keck Interferometer," ApJ 592, pp. L83-L86, Aug. 2003. 